\title{
Publisher Correction: Emergent mystery in the Kondo insulator samarium hexaboride
}

\author{
Lu Li(D), Kai Sun (1), Cagliyan Kurdak and J. W. Allen
}

Nature Reviews Physics (2020) https://doi.org/10.1038/s42254-020-0210-8 Published online 05 August 2020

In the original version of this manuscript, the graph in Fig 1a was incorrectly rendered, and two traces were not clearly visible. Panels $\mathrm{c}$ and $\mathrm{d}$ in Fig 3 were mislabelled in the caption and in the text. A reference in the caption of Fig 6 linked to the wrong citation. Finally, in the list of highlighted references, the text referred to a singular 'surface state' instead of the plural 'surface states', which is more accurate. These have been corrected in the HTML and PDF versions of the manuscript.

https://doi.org/10.1038/s42254-020-0234-0 I Published online 14 August 2020

(c) Springer Nature Limited 2020 